\section{Personalization of $\mathrm{T}$ cell production for cellular immunotherapy}

Dennis Jinglun Yuan ${ }^{1}$, Shuai Shao ${ }^{1}$, Joanne H Lee ${ }^{1}$, Stacey M Fernandes $^{2}$, Jennifer R Brown ${ }^{3}$, and Lance C Kam ${ }^{1}$

${ }^{1}$ Columbia University; ${ }^{2}$ Dana Farber Cancer Institute; ${ }^{3}$ Harvard Medical School

OBJECTIVES/GOALS: Utilize polymer-based fiber scaffolds and machine learning methods applied to patient biomarker data to enhance and personalize $\mathrm{T}$ cell expansion and production for $\mathrm{T}$ cell therapy in chronic lymphocytic leukemia. METHODS/STUDY POPULATION: Scaffolds are 1) generated from a co-polymer blend of PDMS and PCL with controlled fiber diameters and pore size, 2) coated with activating antibodies to CD3 and CD28, and 3) used to stimulate $\mathrm{T}$ cells from both healthy donors and CLL patients. CLL patients have pre-annotated mutation burdens and clinical biomarkers. T cell populations will be analyzed for exhaustion markers and phenotypes before, during, and after expansion. Cell functionality will be measured by cytokine secretion, cell cycle analysis, and fold expansion, with respect to platform parameters, and analyzed with inputs of disease markers and exhaustion profile of isolated $\mathrm{T}$ cells using regression and random forest classifiers. RESULTS/ ANTICIPATED RESULTS: We previously showed that engineering the mechanical rigidity of activating substrates can enhance and rescue $\mathrm{T}$ cell expansion from exhausted populations. Now we aim to study a broader range of compositions and geometry of scaffolds with respect to capacity to expand CLL T cells. Preliminary data with fiber diameters ranging from $300 \mathrm{~nm}$ to $6 \mathrm{um}$ confirm the effect of geometry in modulating expansion. A biorepository of $\mathrm{T}$ cells from 80 CLL patients have been isolated concurrently. Anticipated results include correlating exhaustion profile of $\mathrm{T}$ cells with clinical biomarkers and identifying markers associated with expansion on panel of platform parameters. DISCUSSION/SIGNIFICANCE OF IMPACT: $\mathrm{T}$ cell therapy has shown particular promise in treating blood cancers, yet significant percentage of $\mathrm{T}$ cells isolated from patients undergoing treatments are unresponsive to activation. A powerful tool is to predict if and how patient $\mathrm{T}$ cells can be robustly expanded on a personalized approach.

4081

Quantifying pH buffering capacity and kinetics of tumor and healthy tissue to understand and exploit differences in biology

A. Colleen Crouch ${ }^{1}$, Emily A. Thompson ${ }^{2}$, Mark D. Pagel ${ }^{2}$, and Erik N.K. Cressman ${ }^{2}$

${ }^{1}$ The University of Texas Health Science Center at Houston; ${ }^{2}$ University of Texas MD Anderson Cancer Center

OBJECTIVES/GOALS: The purpose of this work is to investigate natural buffering capacity of liver tissue and tumors, to understand and exploit differences for therapy. Using this work, we will determine the concentrations of reagents (acids or bases) used in ablation treatment to optimize treatment by increasing tumor toxicity and minimizing healthy tissue toxicity. METHODS/STUDY POPULATION: For this preliminary study, two methods will be used: benchtop $\mathrm{pH}$ experiments ex vivo and non-invasive imaging using acidoCEST MRI in vivo. For ex vivo, two types of tissues will be tested: non-cancerous liver and tumor tissue from HepG2 inoculated mice $(n=10)$. After mice are euthanized, $\mathrm{pH}$ will be measured in tissue homogenates at baseline and then the homogenates will be placed in either acidic (acetic acid) or basic (sodium hydroxide) solutions with varied concentrations $(0.5-10 \mathrm{M})$ and time recorded until $\mathrm{pH}$ returns to baseline. For in vivo imaging, Mia PaCA-2 flank model mice $(\mathrm{n}=10)$ will be imaged with acidoCEST MRI to quantify $\mathrm{pH}$ at baseline. Mice will then be injected intratumorally with (up to $100 \mu \mathrm{L}$ of) acid or base at increasing concentrations and imaged to quantify $\mathrm{pH}$ changes in the tumor. RESULTS/ ANTICIPATED RESULTS: For this study, buffering capacity is defined as the concentration threshold for which tissue can buffer $\mathrm{pH}$ back to within normal range. Non-cancerous tissue is likely to buffer a wider range of concentrations compared to tumor tissue. From the benchtop experiment, comparison of time-to-buffer will be made for each concentration of acid/base for the two tissue types. AcidoCEST MRI will provide in vivo buffering capacity and potentially demonstrate tumor heterogeneity of buffering capacity. For both experiments, a $\mathrm{pH}$ vs. concentration curve for the two tissue types will allow for comparison of ex vivo to in vivo experiments, which will differentiate contributions of local tissue buffering capacity from the full body's natural bicarbonate buffer system that depends on respiration and blood flow. DISCUSSION/SIGNIFICANCE OF IMPACT: The $\mathrm{pH}$ of the body must be maintained within a narrow range. With cancer, impairment in regulation of tumor metabolism causes acidosis, lowering extracellular $\mathrm{pH}$ in tumors. It remains unclear if $\mathrm{pH}$ plays a role in local recurrence or tumor toxicity. This work will determine if acidoCEST MRI can measure deliberate alteration of $\mathrm{pH}$ and how this change affects biology.

4335

Role of PSD95 and nNOS interaction in gene regulation following fear conditioning and implications for molecular mechanisms underlying PTSD

Jheel Patel ${ }^{1}$, Erik Dustrude, Melissa Haulcomb, Liangping Li, Guanglong Jiang, Yunlong Liu, Yvonne Lai, Andrei Molosh, and Anantha Shekhar, MD, PhD ${ }^{1}$

${ }^{1}$ Indiana University School of Medicine

OBJECTIVES/GOALS: Normal fear learning produces avoidance behavior that promotes survival, but excessive and persistent fear after trauma can lead to development of phobias and post-traumatic stress disorder (PTSD). Our goal is to understand the mechanism and identify novel genetic targets underlying fear responses. METHODS/STUDY POPULATION: Involvement of the amygdala in fear acquisition is well established and requires activation of N-methyl-D-aspartic acid receptors (NMDARs). At a cellular level, NMDAR activation leads to production of nitric oxide (NO) by a process mediated by interaction between postsynaptic density protein 95 (PSD95) and neuronal nitric oxide synthase (nNOS). To elucidate mechanisms underlying the role of the PSD95-nNOS-NO pathway in conditioned fear, here we use rodent behavioral paradigms, pharmacological treatment with a small molecular PSD95-nNOS inhibitor, co-immunoprecipitation, Western blotting, and RNA-sequencing. RESULTS/ANTICIPATED RESULTS: We show that fear conditioning enhances the PSD95-nNOS interaction and that the small-molecule ZL006 inhibits this interaction. Treatment with ZL006 also attenuates rodent cued-fear consolidation and prevents fear-mediated shifts in glutamatergic receptor and current densities in the basolateral amygdala (BLA). With RNA-sequencing, expression of 516 genes was altered in the BLA following fear expression; of these genes, 83 were restored by systemic ZL006 treatment. Network data and gene ontology enrichment analysis with Ingenuity Pathway Analysis and DAVID software found that cell-cell interaction, cognition-related pathways, and insulin-like growth factor binding 